(C) 2015 IEEE. Personal use of this material is permitted. Permission from IEEE must be obtained for all other uses, in any current or future media, including reprinting/republishing this material for advertising or promotional purposes, creating new collective works, for resale or redistribution to servers or lists, or reuse of any copyrighted component of this work in other works

\title{
Linear quadratic control of LPV systems using static and shifting specifications
}

\author{
Damiano Rotondo, Vicenç Puig, Fatiha Nejjari
}

\begin{abstract}
This paper extends some recent results about linear quadratic control (LQC) using linear matrix inequalities (LMIs) to linear parameter varying (LPV) systems. At first, static specifications, where the weighting matrices are constant, are considered. Later, the concept of shifting linear quadratic control (SLQC), where some varying parameters are introduced and used to schedule not only the controller, but the weighting matrices too, is considered. A numerical example is used to illustrate the application of the proposed theory.
\end{abstract}

Keywords: Linear parameter-varying systems, optimal control.

\section{INTRODUCTION}

Linear quadratic control (LQC) has played an important role in modern control theory [1]. Since the pioneer work by Kalman [2], many researchers have investigated this problem [3], [4]. Historically, this problem was first investigated in the case of white Gaussian noise [5], [6], and has been recently extended to arbitrary correlated noise [7]. The study of LQC still motivates the interest of the research community, as testified by the big number of works that are published about this subject [8]-[12].

In the last decades, gain-scheduling control techniques have consolidated as an efficient answer to analysis and synthesis problems for nonlinear systems [13]. The strength of these techniques consists in the fact that the properties of the nonlinear systems are checked on the basis of a collection of linear systems, that is also used for designing the controller. This is done in a divide and conquer fashion so that well established linear methods can be applied to nonlinear problems.

Linear parameter varying (LPV) systems were introduced by Shamma [14] to distinguish such systems from linear time invariant (LTI) and linear time varying (LTV) ones [15]. More specifically, LPV systems are a particular class of LTV systems, where the time-varying elements depend on measurable parameters that can vary over time [16]. The LPV framework has proved to be suitable for controlling nonlinear systems by embedding the nonlinearities in the varying parameters, that will depend on some endogenous signals, e.g. states, inputs or outputs. In this case, the system is referred to as quasi- $L P V$, to make a further distinction with

This work has been funded by the Spanish Ministry of Science and Technology through the projects CICYT SHERECS (ref. DPI2011-26243) and CICYT ECOCIS (ref. DPI2013-48243-C2-1-R), by AGAUR through the contract FI-DGR 2014 (ref. 2014FI_B1 00172) and by the DGR of Generalitat de Catalunya (SAC group Ref. 2014/SGR/374).

D. Rotondo, V. Puig and F. Nejjari are with Automatic Control Department, Universitat Politècnica de Catalunya (UPC), Rambla de Sant Nebridi, 11, 08222 Terrassa, Spain. e-mail: \{damiano.rotondo, fatiha.nejjari,vicenc.puig\}@upc.edu respect to pure LPV systems, where the varying parameters only depend on exogenous signals [17]. The LPV paradigm has evolved rapidly in the last two decades, and has been applied successfully to a big number of applications [18], e.g. airplanes [19], robots [20], unmanned aerial vehicles (UAVs) [21], vehicle suspensions [22] and wind turbines [23].

Recently, solutions to the LQC problem have been proposed in terms of linear matrix inequalities (LMIs) [24], [25]. LMIs are a standard formulation for solving complicated control problems very efficiently, and with a remarkable degree of simplicity. Also, the conditions provided for LTI systems can be easily extended to deal with LPV systems such that, as a result, they are useful to analyse and design control laws for nonlinear systems too.

The goal of this paper is to use the recent results obtained in [24] to propose an LQC design procedure for LPV systems, in both the continuous-time and the discrete-time cases. At first, static specifications, where the weighting matrices are constant, are considered. Later, the case of shifting specifications, where some varying parameters are introduced and used to schedule not only the controller, but the weighting matrices too, is considered. Shifting specifications, introduced in [26], can be of practical interest for all situations where some online variations of performance could be desirable, e.g. control of systems with saturation nonlinearities [27], graceful performance degradation for active fault-tolerant control [28] and actuator health degradation avoidance [29]. In the case of LQC, by considering shifting specifications, the concept of shifting linear quadratic control (SLQC) is obtained.

This paper is structured as follows. Section II extends results about LQC of LTI systems to the case of LQC of LPV systems. Shifting specifications are considered in Section III, thus obtaining SLQC of LPV systems. A numerical example is used to illustrate the application of the proposed theory in Section IV. Finally, the main conclusions are summarized in Section V.

Notation: If a matrix $M \in \mathrm{R}^{n \times n}$ is symmetric, then $M \in$ $\mathrm{S}^{n \times n}$. A matrix $M \in \mathrm{S}^{n \times n}$ is said positive definite $(M>-O)$ if all its eigenvalues are positive, and negative definite $(M<O)$ if all its eigenvalues are negative. Similarly, the matrix $M$ is said positive semi-definite if all its eigenvalues are nonnegative $(M t O)$, and negative semi-definite $(M: j O)$ if all its eigenvalues are non-positive. For brevity, symmetric elements in a matrix are denoted by $*$ and $M+M^{T}$ will be indicated as $\mathrm{He}\{M\}$. 


\section{LQC FOR LPV SYSTEMS}

\section{A. Continuous-time LQC}

The results presented hereafter extend to the continuoustime LPV case the ones obtained by [24] for the continuoustime LTI case. Given an LPV system, defined by:

$$
\dot{x}(t)=A(\theta(t)) x(t)+B(\theta(t)) u(t)
$$

where $x \in \mathrm{R}^{n_{x}}$ is the state vector, $u \in \mathrm{R}^{n_{u}}$ is the input vector, and $A(\theta) \in \mathrm{R}^{n_{x} \times n_{x}}$ and $B(\theta) \in \mathrm{R}^{n_{x} \times n_{u}}$ are matrix functions of a vector of varying parameters $\theta(t) \in \Theta \subset \mathrm{R}^{n_{\theta}}$, with initial condition $x(0)=x_{0}$ specified, find a state feedback control law:

$$
u(t)=K(\theta(t)) x(t)
$$

such that the following quadratic criterion:

$$
J={ }_{0}^{r^{\infty}}{ }^{\mathrm{i}}(t)^{T} Q x(t)+u(t)^{T} R u(t) d t
$$

is minimized, where $Q=H^{T} H \boldsymbol{t} O$ and $R>-O$, with $H \in$ $\mathrm{R}^{n_{q} \times n_{x}}, n_{q}=\operatorname{rank}(Q)$, and $R \in \mathrm{S}^{n_{u} \times n_{u}}$.

A practical approach for minimizing $J$ is to search for a control law (2) which guarantees that the criterion $J$ is below some number $\mathrm{Y}[30]$.

Let us introduce the Lyapunov function $V(x(t))=$ $x(t)^{T} P x(t)$, with $P>-O, P \in \mathrm{S}^{n_{x} \times n_{x}}$, such that:

$$
\begin{gathered}
V\left(x_{0}\right)=x_{0}^{T} P x_{0}<\mathrm{Y} \\
\dot{V}(x(t))+x(t)^{T} Q x(t)+u(t)^{T} R u(t)<0
\end{gathered}
$$

that, taking into account (2), becomes:

$$
\dot{V}(x(t))+x(t)^{T} Q+K(\theta(t))^{T} R K(\theta(t)) \quad x(t)<0
$$

which leads to [24]:

$$
J={ }_{0}^{r^{\infty} r} x(t)^{T}{ }^{\perp} Q+K(\theta(t))^{T} R K(\theta(t)) \quad x(t) \quad d t<V\left(x_{0}\right)<y
$$

By recalling that:

$$
\dot{V}(x(t))=\dot{x}(t)^{T} P x(t)+x(t)^{T} P \dot{x}(t)
$$

and taking into account (1)-(2), the following matrix inequality can be obtained from (5) ${ }^{1}$ :

$$
\begin{array}{rl}
(A(\theta)+B(\theta) K(\theta))^{T} & P+P(A(\theta)+B(\theta) K(\theta)) \\
& +Q+K(\theta)^{T} R K(\theta)<O \quad \forall \theta \in \Theta
\end{array}
$$

Then, taking into account the results about dual matrix inequalities obtained by [31], it can be shown that (9) is equivalent to:

$$
\begin{aligned}
P(A(\theta)+B(\theta) K(\theta))^{T}+(A(\theta)+B(\theta) K(\theta)) P & \\
& +P Q P+P K(\theta)^{T} R K(\theta) P<O \quad \forall \theta \in \Theta
\end{aligned}
$$

\footnotetext{
${ }^{1}$ The symbol $\theta$ is used to indicate a generic value of the varying parameter, whereas $\theta(t)$ indicates a specified trajectory.
}

Eq. (10), after a change of variable $\Gamma(\theta) \_\mathrm{Q} K(\theta) P$, becomes:

$$
\begin{aligned}
& P A(\theta)^{T}+\Gamma(\theta)^{T} B(\theta)^{T}+A(\theta) P+B(\theta) \Gamma(\theta) \\
& \left.+{ }^{(} \begin{array}{lll}
P H^{T} & \Gamma(\theta)^{T}
\end{array}\right)\left({ }_{O}^{I}{ }_{R}^{O}{ }_{\Gamma(\theta)}^{H P}\right)
\end{aligned}
$$

By applying Schur complements [32], (4) and (11) can be reshaped as:

$$
\begin{aligned}
& \begin{array}{cc}
{ }_{Y} & x_{0}^{T} \\
x_{0} & P
\end{array}>-0
\end{aligned}
$$

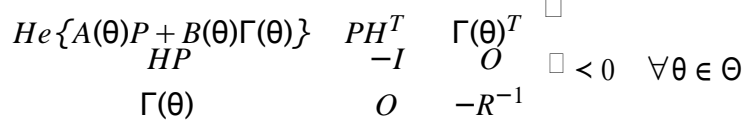

The state feedback controller gain is thus obtained by solving the LMIs (12)-(13), and letting $K(\theta)=\Gamma(\theta) P^{-1}$.

However, from a practical point of view, (13) is useless because it corresponds to an infinite number of constraints. Under the assumption that $B$ is constant, and that $A(\theta)$ is polytopic, i.e. it satisfies the following property:

$$
A(\theta(t))=\sum_{i=1}^{N} \alpha_{i}(\theta(t)) A_{i}
$$

with:

$$
\sum_{i=1}^{N} \alpha_{i}(\theta(t))=1 \quad \alpha_{i}(\theta(t)) \geq 0
$$

it is possible to choose the control law (2) to be polytopic as well:

$$
u(t)=\sum_{i=1}^{N} \alpha_{i}(\theta(t)) K_{i} x(t)
$$

and to reduce (13) to a finite number of LMIs written at the vertices of the polytope, as follows:

$$
\begin{array}{ccc}
H e\left\{A_{i} P+B \Gamma_{i}\right\} & P H^{T} & \Gamma_{i}^{T} \\
H P & -I & O \\
\Gamma_{i} & O & -R^{-1}
\end{array}
$$

Notice that the assumption of a constant $B$ is not restrictive, since in the case of a varying $B(\theta)$, a prefiltering of the input $u(t)$ would lead to obtain a new system with a constant matrix $\tilde{B}$ [33]. More specifically, for the system (1), let us define a new control input $\tilde{u}(t)$ such that:

$$
\begin{gathered}
\dot{x}_{u}(t)=A_{u}(\theta(t)) x_{u}(t)+B_{u} \tilde{u}(t) \\
u(t)=C_{u} x_{u}(t)
\end{gathered}
$$

with $A_{u}(\theta(t))$ stable. Then, the resulting LPV system would be:

$$
\begin{gathered}
\dot{x}_{\dot{x}(t)} \\
\dot{x}_{u}(t)
\end{gathered}=\tilde{A}(\theta(t))\left(\begin{array}{l}
x(t) \\
x_{u}(t)
\end{array} \quad+\tilde{B} \tilde{u}(t)\right.
$$

with:

$$
\begin{array}{cc}
A(\theta(t)) & B(\theta(t)) C_{u} \\
O & A_{u}(\theta(t))
\end{array} \quad \tilde{B}=\begin{gathered}
O \\
B_{u}
\end{gathered}
$$

Then, if it is desired to minimize the value of $\gamma$, the following optimization problem should be solved:

$$
\min _{P, \Gamma_{i}, i=1, \ldots, N} \mathrm{Y}
$$


subject to (12) and (17).

\section{B. Discrete-time $L Q C$}

Similarly to the continuous-time case, the results presented hereafter extend to the discrete-time LPV case the ones obtained by [24] for the discrete-time LTI case. Given the LPV system:

$$
x(k+1)=A(\theta(k)) x(k)+B(\theta(k)) u(k)
$$

and the quadratic criterion:

$$
J=\sum_{k=0}^{\infty}\left(x(k)^{T} Q x(k)+u(k)^{T} R u(k)\right.
$$

minimize $J$ by means of the state feedback control law:

$$
u(k)=K(\theta(k)) x(k)
$$

In this case, the Lyapunov function is chosen as $V(x(k))=$ $x(k)^{T} P x(k), P>-O, P \in \mathrm{S}^{n_{x} \times n_{x}}$, such that (4) and:

$$
V(x(k+1))-V(x(k))+x(k)^{T} Q x(k)+u(k)^{T} R u(k)<0
$$

Then, the following is obtained:

$$
\begin{aligned}
(A(\theta)+B(\theta) K(\theta))^{T} P(A(\theta)+B(\theta) K(\theta))-P & \\
+Q+K(\theta)^{T} R K(\theta) & <O \quad \forall \theta \in \Theta
\end{aligned}
$$

that, recalling $Q=H^{T} H$, and after the change of variable $\Gamma(\theta) \_\mathrm{Q} K(\theta) P$, leads to the matrix inequality [31]:

$$
\begin{aligned}
( \\
P A(\theta)^{T}+\Gamma(\theta)^{T} B(\theta)^{T}{ }^{T} P^{-1}(A(\theta) P+B(\theta) \Gamma(\theta))-P \\
+P H^{T} H P+\Gamma(\theta)^{T} R \Gamma(\theta)<O \quad \forall \theta \in \Theta
\end{aligned}
$$

By applying Schur complements [32], (4) and (28) are reshaped into (12) and:

$$
\begin{aligned}
& \begin{array}{crrcll}
-P & * & P H^{T} & \Gamma(\theta)^{T} & & \\
A(\theta) P+B(\theta) \Gamma(\theta) & -P & O & O & \square
\end{array} \quad \forall \theta \in \Theta \\
& \begin{array}{llll}
H P & O & -I & O
\end{array} \\
& \Gamma(\theta) \quad O \quad O \quad-R^{-1}
\end{aligned}
$$

Also in this case, under the assumptions of $B$ constant (cases with varying $B(\theta)$ are treated in the same way as the continuous-time case, using the filter proposed by [33]) and

$A(\theta)$ and $K(\theta)$ polytopic, it is possible to reduce (29) to the following finite number of LMIs:

$$
\begin{aligned}
& -P \quad P A^{T}+\Gamma^{T} B^{T} \quad P H^{T} \quad \Gamma^{T} \\
& A_{i} P+B \Gamma_{i} \quad{ }^{i}-P^{i} \\
& \text { HP O } \\
& \begin{array}{ccc}
O & \dot{O} & \square \\
-I & O & \square<O \quad i=1, \ldots, N
\end{array} \\
& \Gamma_{i} \quad O \\
& O \quad-R^{-1}
\end{aligned}
$$

and obtain the vertex controller gains as $K_{i}=\Gamma_{i} P^{-1}$

Then, if it is desired to minimize the value of $\gamma$, the

optimization problem (22) subject to (12) and (30) should be solved.

\section{SHIFTING LQC FOR LPV SYSTEMS}

In this section, we consider the problem of designing an LPV state-feedback control law:

$$
u(\mathrm{~T})=K_{s}(\theta(\mathrm{T}), p(\mathrm{~T})) x(\mathrm{~T})
$$

where $\mathbf{T}=t$ in the continuous-time case, $\mathbf{T}=k$ in the discretetime case, and $p(\mathrm{~T}) \in \Pi \subset \mathrm{R}^{n_{p}}$ is a vector of scheduling parameters defined in the following and used to achieve the shifting linear quadratic control (SLQC).

Remark 1: Despite in this paper the problem of controller design using SLQC is considered for the case of LPV systems, the proposed method is useful for LTI systems too. In this case, the controller is scheduled by means of the vector of parameters $p(\mathrm{~T})$, such that, even though the plant to be controlled is LTI, the overall system is LPV and the mathematical reasoning developed hereafter can be applied. The reason to do so is that in this way the performance of the closed-loop system can be varied in time according to some criterium, e.g. energetic issues.

\section{A. Continuous-time SLQC}

Given the continuous-time LPV system (1), the SLQC problem aims at designing the controller $K_{s}(\theta(t), p(t))$ in (31) such that for $p(t)=p$ it minimizes:

$$
J(p)={ }_{0}^{\mathrm{r}^{\infty}}{ }_{x(t)^{T}} Q(p) x(t)+u(t)^{T} R(p) u(t)^{\mathrm{I}} d t
$$

Notice that since $Q$ and $R$ are scheduled by $p$, and the controller as well, the characteristics of optimality in the sense of the considered criterion can be varied online. Thus, the performances of the closed-loop system can be changed online, being this the main feature of the SLQC that distinguishes it from the classical LQC.

Remark 2: The SLQC specification demands the optimality of $J(p)$ for $p(t)=p$, i.e. for a constant value of $p(t)$. It is worth highlighting that the proposed solution leads to sub-optimality, because of the minimization of the upper bound $\mathrm{y}$ instead of the performance index $J(p)$. However, this approach has the strong advantage of obtaining design

conditions that can be solved very efficiently from a computational point of view. It is also worth noticing that, even if the SLQC is defined for $p(t)=p$, the controller designed using this specification provides good performances also in the case of varying $p(t)$, as shown later in the example.

Then, by guaranteeing that $J(p)$ is below $\mathrm{\gamma}$, and using the Lyapunov function $V(x(t))=x(t)^{T} P x(t)$, with $P>-O$, $P \in \mathrm{S}^{n_{x} \times n_{x}}$, by considering $Q(p)=H(p)^{T} H(p) \forall p \in \Pi$, and applying the reasoning described in Section II-A, (12) and:

$$
\begin{array}{cccc}
H e\{A(\theta) P+B(\theta) \Gamma(\theta, p)\} & P H(p)^{T} & \Gamma(\theta, p)^{T} & \\
H(p) P & -I & O & \forall \in \Theta \\
\Gamma(\theta, p) & O & -R(p)^{-1} & \\
& & & \forall p \in \Pi
\end{array}
$$

are obtained. 
Since (33) represents infinite constraints, the assumptions that $B$ is constant, and that:

$$
\begin{gathered}
H(p) \\
R(p)^{-1}
\end{gathered} \quad=\sum_{j=1}^{P} \Pi_{j}(p) \stackrel{C_{\tilde{R}_{j}^{j}}^{H_{j}}}{\underset{ }{H^{\prime}}} \quad \forall p \in \Pi
$$

with:

$$
\sum_{j=1}^{P} \Pi_{j}(p)=1 \quad \Pi_{j}(p) \geq 0
$$

is done. Then, by choosing $K_{s}(\theta(t), p(t))$ as:

$$
K_{s}(\theta(t), p(t))=\sum_{i=1}^{N} \alpha_{i}(\theta(t)) \sum_{j=1}^{P} \Pi_{j}(p) K_{i j}
$$

(33) cąn be reducęd to ${ }^{2}$ :

$$
\begin{aligned}
& H e^{\{} A_{i} P+B \Gamma_{i j} \quad P H_{j}^{T} \quad \Gamma_{i j}^{T}
\end{aligned}
$$

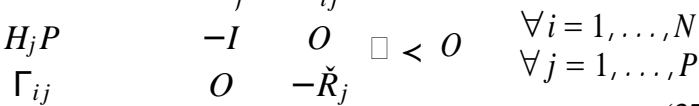

and the vertex controller gains can be calculated as $K_{i j}=$ $\Gamma_{i j} P^{-1}$. Then, (22) can be minimized subject to (12) and (37).

\section{B. Discrete-time SLQC}

Similarly to the continuous-time case, given the discretetime LPV system (23), the SLQC problem is to design the controller $K_{s}(\theta(k), p(k))$ in (31) such that for $p(k)=p$ it minimizes:

$$
J(p)=\sum_{k=0}^{\infty}\left(x(k)^{T} Q(p) x(k)+u(k)^{T} R(p) u(k)\right.
$$

Remark 3: Considerations similar to the ones made for continuous-time systems in Remark 2 are valid for discretetime systems as well.

By using the Lyapunov function $V(x(k))=x(k)^{T} P x(k)$, with $P>-O, P \in S^{n_{x} \times n_{x}}$, by considering $Q(p)=$ $H(p)^{T} H(p) \forall p \in \Pi$, and applying the reasoning described in Section II-B, (12) and:

$$
\begin{array}{crccl}
-P & * & P H(p)^{T} & \Gamma(\theta, p)^{T} & \\
A(\theta) P+B(\theta) \Gamma(\theta, p) & -P & O & O & \square \\
H(p) P & O & -I & O & \square<O \\
\Gamma(\theta, p) & O & O & -R(p)^{-1} &
\end{array}
$$

which must hold $\forall \theta \in \Theta$ and $\forall \pi \in \Pi$, are obtained.

Then, if $B$ is constant, and (34)-(36) hold, (39) is equivalent to:

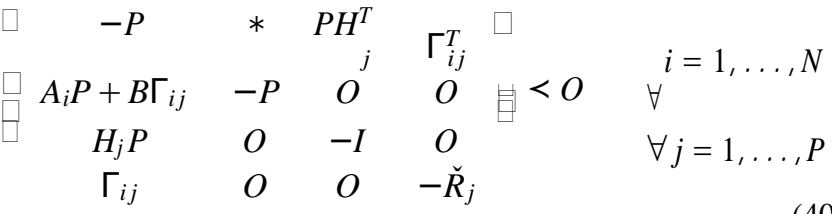

such that the vertex controller gains can be calculated as $K_{i j}=\Gamma_{i j} P^{-1}$. Then, (22) can be minimized subject to (12) and (40).

\footnotetext{
${ }^{2} \mathrm{~A}$ formal proof of this fact is not given in this work, due to space limitation. However, it follows a reasoning similar to the one provided in [26].
}

\section{EXAMPLE}

Consider a discrete-time LPV system as in (23), with matrices defined as follows:

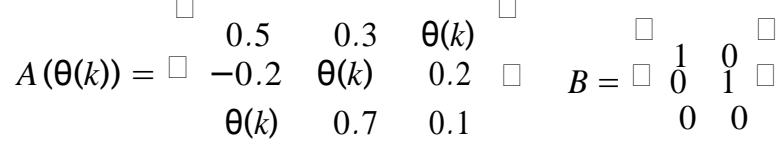

where $\theta(k) \in[-0.2,0.2]$, and consider the problem of designing controller gains $K(\theta(k))$ in (25) such that, starting from $x_{0}=[1,0,0]^{T}$, they minimize the quadratic criteria, defined as in (38), in the following three cases:

- Case A, $K_{A}(\theta(k))$

$$
\left.J_{A} \Rightarrow Q_{A}=\begin{array}{ccc}
1 & 0 & 0 \\
0 & 1 & 0 \\
0 & 0 & 1
\end{array} \square \quad R_{A}=\begin{array}{cc}
C_{1} & 0 \\
0 & 1
\end{array}\right)
$$

- Case B, $K_{B}(\theta(k))$

$$
\left.J_{B} \Rightarrow Q_{B}=\begin{array}{rrr}
1 & 0 & 0 \\
0 & 10 & 0 \\
0 & 0 & 1
\end{array} \square \quad R_{B}=\begin{array}{cc}
10 & 0 \\
0 & 1
\end{array}\right)
$$

- Case $C, K_{C}(\theta(k))$

$$
J_{C} \Rightarrow Q_{C}=\begin{array}{ccc}
1 & 0 & 0 \\
0 & 1 & 0 \\
0 & 0 & 10
\end{array} \quad \square \quad R_{C}=\begin{array}{cc}
\left(\begin{array}{c} 
\\
10
\end{array}\right. & 0 \\
0 & 10
\end{array}
$$

The procedure described in Section II-B provides the controller gains with the following bounds on the quadratic criterion: $\mathrm{Y}_{A}=1.2476, \mathrm{Y}_{B}=1.4673$ and $\mathrm{Y}_{C}=2.4634$. YALMIP toolbox [34] with SeDuMi solver [35] has been used to find a feasible solution to the LMIs.

Later, the following varying weighting matrices in the quadratic criterion $J(p)$, defined as in (38), have been considered:

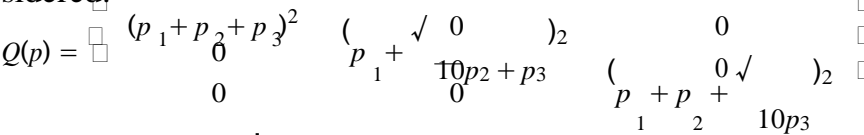

$$
\begin{aligned}
& R(p)=\begin{array}{ccc}
\text { I } & & \\
10 p \quad 10 p & 0 \\
1+p_{2}+p_{3} & \frac{10}{10 p_{1}+10 p_{2}+p_{3}}
\end{array}
\end{aligned}
$$

with $p_{1}, p_{2}, p_{3} \in[0,1]$.

Notice that $Q(p)$ and $R(p)$ correspond to:

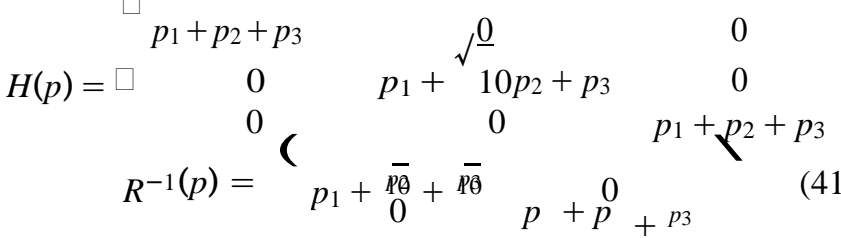

$$
\begin{aligned}
& \begin{array}{lll}
1 & 2 & \overline{10}
\end{array}
\end{aligned}
$$

which can be easily expressed as (34) choosing $\Pi_{1}=p_{1}, \Pi_{2}=$ $p_{2}$ and $\pi_{3}=p_{3}$, under the constraint that $p_{1}+p_{2}+p_{3}=1$, with the relevant feature that the values of $H(p)$ and $R^{-1}(p)$ in the cases $\left[p_{1}, p_{2}, p_{3}\right]=[1,0,0],\left[p_{1}, p_{2}, p_{3}\right]=[0,1,0]$ and $\left[p_{1}, p_{2}, p_{3}\right]=[0,0,1]$ correspond to $H_{A}$ and $R_{A}^{-1}, H_{B}$ and $R_{B}^{-1}$,

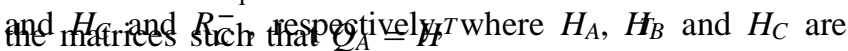
$H_{C}^{T} H_{C}$

$$
{ }_{A} H_{A}, Q_{B}=H_{B} H_{B} \text { and } Q_{C}=
$$




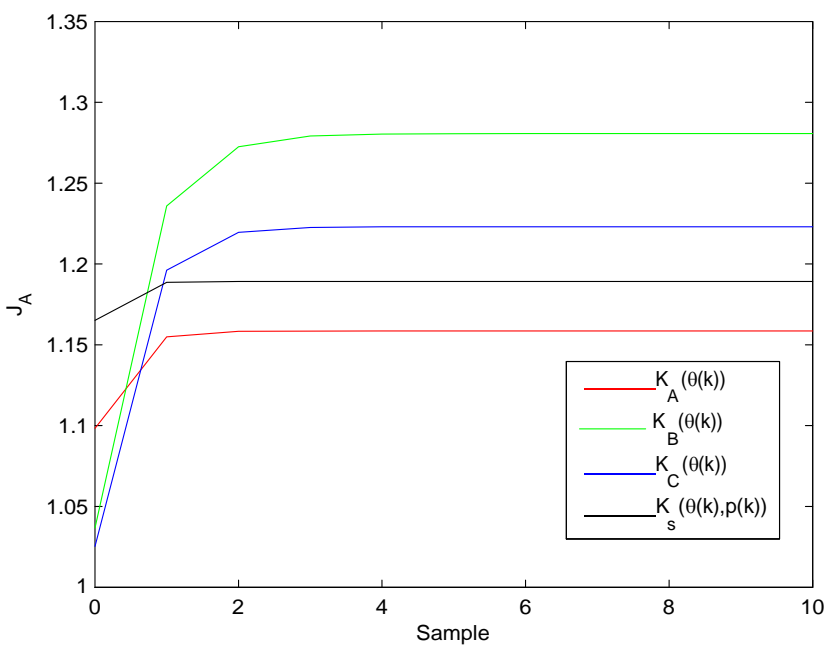

Fig. 1. Performance index $J_{A}$ obtained with the designed controllers.

Then, a controller $K_{s}(\theta(k), p(k))$ has been obtained using the procedure described in Section III-B with a bound $\mathrm{Y}=$ 2.4726 .

Figs. 1-3 show the evolutions of the performance indices $J_{A}, J_{B}$ and $J_{C}$ using the designed controllers. As expected, $K_{A}, K_{B}$ and $K_{C}$ obtain the best performance when using the index $J_{A}, J_{B}$ and $J_{C}$, respectively. Also, each one outperforms $K_{s}$ in the corresponding case, even though it can be observed that the performances obtained by $K_{s}$ are only slightly worse than the best achieved performance.

Finally, in order to complete the comparison, let us consider a varying performance quadratic criterion $J(p)$, where at each sample the values of the elements of the weighting matrices $Q(p)$ and $R(p)$ vary as shown in Fig. 4.

Fig. 5 shows that in this case the best performance is obtained by $K_{s}$. This is due to the fact that, unlike the other controllers that were designed using fixed weighting matrices, $K_{s}$ has been designed using the SLQC concept, that allows to consider the case of a varying specification into the design step.

\section{CONCLUSIONS}

This paper has presented an LMI-based approach for designing linear quadratic controllers in the case of LPV systems, extending results that were obtained recently in the LTI case. Also, the concept of shifting linear quadratic control has been introduced. The feature of this technique that distinguishes SLQC from the standard LQC is that the weighting matrices of the quadratic performance criterion are assumed to vary, and their variations are used to schedule the controller. The theory has been developed for both the continuous-time and the discrete-time cases, and a discretetime numerical examples has been used to illustrate the application of the proposed theory. The results have shown that in the cases of fixed weighting matrices, the controller that has been designed to be optimal for the specific weighting matrix taken into consideration outperforms the other controllers.

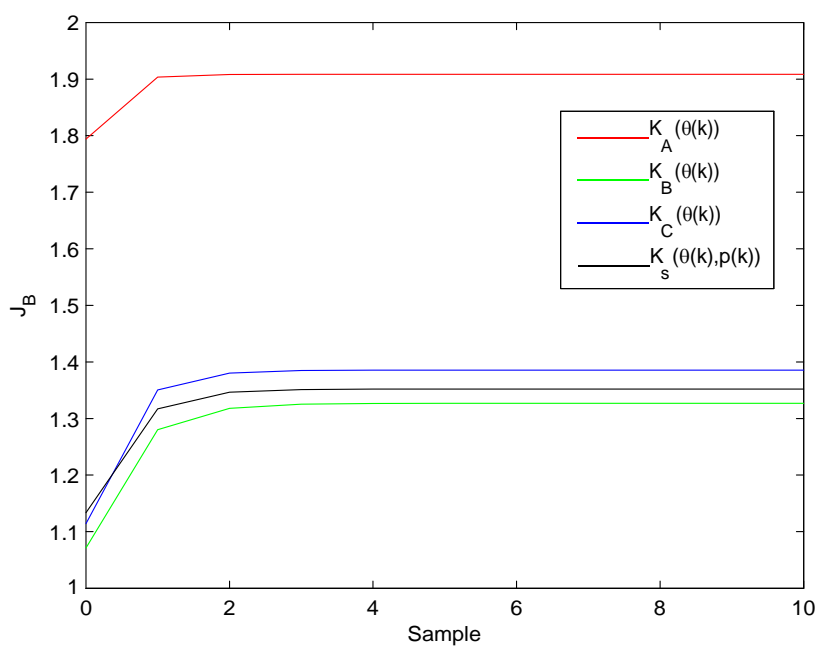

Fig. 2. Performance index $J_{B}$ obtained with the designed controllers.

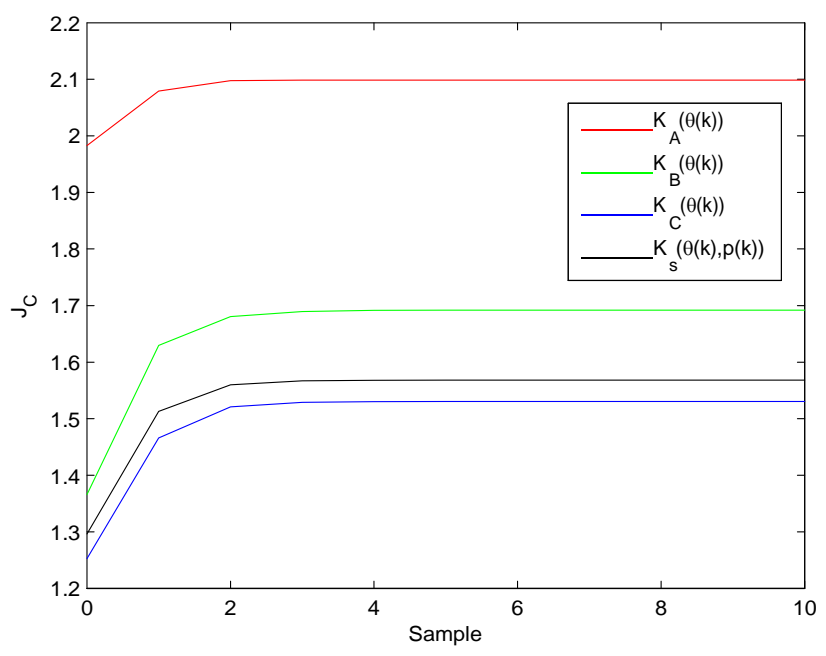

Fig. 3. Performance index $J_{C}$ obtained with the designed controllers.

However, in these cases the SLQC controller performances are only slightly worse than the best obtained performances, and when a varying performance index is considered, it proves to be the best one.

\section{REFERENCES}

[1] X. Liu, Y. Li, and W. Zhang, "Stochastic linear quadratic optimal control with constraint for discrete-time systems," Applied Mathematics and Computation, vol. 228, no. 1, pp. 264-270, 2014.

[2] R. E. Kalman, "Contributions to the theory of optimal control," Boletin de la Sociedad Matematica Mexicana, vol. 5, pp. 102-119, 1960.

[3] B. D. O. Anderson and J. B. Moore, Optimal control linear quadratic methods. Prentice-Hall, New York, 1989.

[4] F. L. Lewis, Optimal control. John Wiley and Sons, New York, 1986.

[5] M. Aoki, Optimization of stochastic systems. New York: Academic Press, 1967.

[6] R. E. Kalman and R. W. Koepcke, "Optimal synthesis of linear sampling control systems using generalized performance indices,” Transactions of the American Society of Mechanical Engineers, vol. 80, pp. 1820-1826, 1958. 


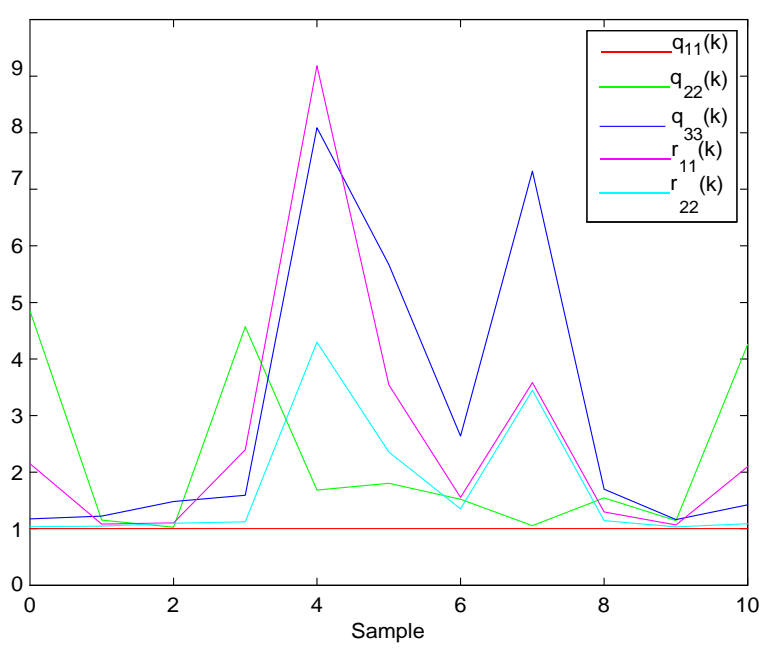

Fig. 4. Elements of the varying weighting matrices $Q(p)$ and $R(p)$.

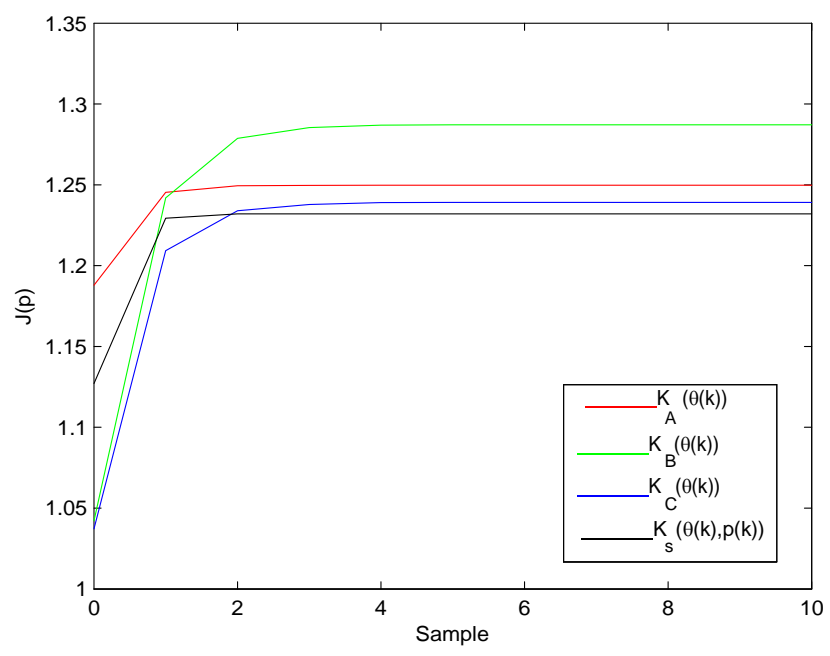

Fig. 5. Performance index $J(p)$ obtained with the designed controllers.

[7] T. E. Duncan and B. Pasik-Duncan, "Discrete time linear quadratic control with arbitrary correlated noise," IEEE Transactions on Automatic Control, vol. 58, no. 5, pp. 1290-1293, 2013.

[8] N. Kumaresan and K. Ratnavelu, "Optimal control for stochastic linear quadratic singular neuro Takagi-Sugeno fuzzy system with singular cost using genetic programming," Applied Soft Computing, vol. 24, pp. 1136-1144, 2014.

[9] M. Haugh and A. E. B. Lim, "Linear-quadratic control and information relaxations," Operations Research Letters, vol. 40, pp. 521-528, 2012.

[10] J. Huang, X. Li, and J. Shi, "Forward-backward linear quadratic stochastic optimal control problem with delay,” Systems and Control Letters, vol. 61, pp. 623-630, 2012.

[11] J. Frédéric Bonnans and F. J. Silva, "Error estimates for the logarithmic barrier method in linear quadratic stochastic optimal control problems," Systems and Control Letters, vol. 61, pp. 143-147, 2012.

[12] A. Gattami, "Generalized linear quadratic control," IEEE Transactions on Automatic Control, vol. 55, no. 1, pp. 131-136, 2010.

[13] D. J. Leith and W. E. Leithead, "Survey of gain-scheduling analysis design,” International Journal of Control, vol. 73, pp. 1001-1025, 2000.
[14] J. S. Shamma, "Analysis and design of gain scheduled control systems,” Ph.D. dissertation, Massachussets Institute of Technology, Department of Mechanical Engineering, advised by M. Athans, 1988.

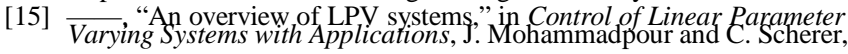
Eds. Springer, 2012.

[16] A. P. White, G. Zhu, and J. Choi, Linear parameter-varying control for engineering applications. Springer-Verlag London Ltd., 2013.

[17] M. Andrés and G. J. Balas, "Development of Linear Parameter Varying Models for Aircraft," Journal of Guidance, Control and Dynamics, vol. 27, no. 2, pp. 218-228, 2004.

[18] C. Hoffmann and H. Werner, "A survey of linear parameter-varying control applications validated by experiments or high-fidelity simulations," IEEE Transactions on Control Systems Technology.

[19] I. Szászi, A. Marcos, G. J. Balas, and J. Bokor, "Linear parametervarying detection filter design for a Boeing 747-100/200 aircraft,” Journal of Guidance, Control, and Dynamics, vol. 28, no. 3, pp. 461470, 2005.

[20] S. Hashemi, H. S. Abbas, and H. Werner, "Low-complexity linear parameter-varying modeling and control of a robotic manipulator," Control Engineering Practice, vol. 20, no. 3, pp. 248-257, 2012.

[21] F. Niel, Y. Ameho, J.-M. Biannic, F. Defa, and C. Bérard, "A novel parameter varying controller synthesis method for quadrotor control," in Proceedings of the AIAA Guidance, Navigation, and Control Conference, 2013.

[22] I. J. Fialho and G. Balas, "Design of nonlinear controllers for active vehicle suspensions using parameter-varying control synthesis," Vehicle Systems Dynamics, vol. 33, no. 5, pp. 351-370, 2000.

[23] F. A. Shirazi, K. M. Grigoriadis, and D. Viassolo, "Wind turbine integrated structural and LPV control design for improved closed-loop performance," International Journal of Control, vol. 85, no. 8, pp. 1178-1196, 2012.

[24] E. Ostertag, Mono- and multivariable control and estimation: Linear, quadratic and LMI methods. Springer-Verlag Berlin Heidelberg, 2011.

[25] G.-R. Duan and H.-H. Yu, LMIs in control systems: analysis, design and applications. CRC Press, Taylor and Francis Group, 2013.

[26] D. Rotondo, F. Nejjari, and V. Puig, "Design of parameter-scheduled state-feedback controllers using shifting specifications," Journal of the Franklin Institute, vol. 352, no. 1, 2015.

[27] F. Wu, K. M. Grigoriadis, and A. Packard, "Anti-windup controller design using linear parameter-varying control methods," International Journal Of Control, vol. 73, pp. 1104-1114, 2000.

[28] Y. Zhang, J. Jiang, and D. Theilliol, "Incorporating performance degradation in fault tolerant control system design with multiple actuator failures," International Journal of Control, Automation, and Systems, vol. 6, no. 3, pp. 327-338, 2008.

[29] A. Khelassi, D. Theilliol, P. Weber, and J.-C. Ponsart, "Fault-tolerant control design with respect to actuator health degradation: An LMI approach," in Proceedings of the International Conference on Control Applications (CCA), 2011.

[30] G. Duc, “Introduction aux LMI,” 2002, lecture notes, Supélec.

[31] R. Goebel, T. Hu, and A. R. Teel, "Dual matrix inequalities in stability and performance analysis of linear differential/difference inclusions," Current Trends in Nonlinear Systems and Control Systems and Control: Foundations and Applications, pp. pp. 103-122, 2006.

[32] J. Schur, "Über Potenzreihen, die im Innern des Einheitskreises beschränkt sind,” Journal für die reine und angewandte Mathematik, vol. 147, pp. 205-232, 1917.

[33] P. Apkarian, P. Gahinet, and G. Becker, "Self-scheduled $H_{\infty}$ Control of Linear Parameter-Varying Systems: A Design Example,” Automatica, vol. 31, no. 9, pp. 1251 - 1261, 1995.

[34] J. Löfberg, "YALMIP : a toolbox for modeling and optimization in MATLAB," in Proceedings of the CACSD Conference, Taipei, Taiwan, 2004.

[35] J. F. Sturm, "Using SeDuMi 1.02, a MATLAB toolbox for optimization over symmetric cones," Optimization Methods and Software, vol. 1112, pp. 625-653, 1999. 